\title{
Diabetes Mellitus Associado com Drogas Antipsicóticas Atípicas: Relato de Caso e Revisão da Literatura
}

\section{apresentação de caso}

\author{
Janice Sepúlveda Reis \\ tiago Alvarenga \\ Pedro Weslley S. do Rosário \\ Patrícia A.f.C. Menezes \\ ROBERTA DOS SANTOS ROCHA \\ SAUlo PuRISCh
}

Serviço de Endocrinologia e Metabologia da Santa Casa de Belo Horizonte, MG

Recebido em 13/03/06 Revisado em 17/09/06 Aceito em 20/09/06

\author{
RESUMO
}

Desde a introdução das medicações antipsicóticas atípicas, iniciando com a clozapina, em 1990, muitos relatos associam essas drogas ao desenvolvimento de diabetes mellitus, entre outros distúrbios metabólicos, assim como abertura da doença como cetoacidose. Relatamos o caso de um paciente de 28 anos, com esquizofrenia, admitido em cetoacidose diabética 1 mês após início da terapia com clozapina, sem relação com ganho de peso, mantendo-se com níveis satisfatórios de glicemia, sem tratamento, após suspensão da droga. Revisamos o assunto, com outros casos relatados até o momento, incluindo a associação de outros antipsicóticos atípicos igualmente envolvidos em distúrbios endócrinos. Objetivamos, com o relato deste caso, aumentar a atenção dos clínicos envolvidos no tratamento dos pacientes portadores de distúrbios psiquiátricos para a possibilidade do surgimento de diabetes durante a terapia, e enfatizar a necessidade de aumento da vigilância e do acompanhamento metabólico desses pacientes. (Arq Bras Endocrinol Metab 2007;51/3:488-493)

Descritores: Cetoacidose diabética; Antipsicóticos atípicos; Obesidade; Dislipidemia

\section{ABSTRACT}

Diabetes Mellitus Associated with Atypical Antipsychotic Medications: Case Report and Review of the Literature.

Since the introduction of atypical antipsychotic medications, starting with clozapine in 1990, many studies have associated these drugs with the development of diabetes among other metabolic disorders, as well as with the onset of the disease as ketoacidosis. We report the case of a 28 -yearold patient with schizophrenia who was admitted with diabetic acidosis 1 month after the beginning of clozapine therapy. No weight gain was reported and the patient maintains satisfactory glycemia levels with no treatment required after discontinuation of the drug. The literature on this subject and cases reported so far are reviewed, including the association of other atypical antipsychotic drugs also involved in endocrine disorders. The objective of this report is to raise the awareness of physicians treating psychiatric patients to the possibility of new-onset diabetes during therapy with atypical antipsychotic drugs and to emphasize the necessity for increased vigilance and close metabolic follow-up of these patients. (Arq Bras Endocrinol Metab 2007;51/3:488-493)

Keywords: Diabetic ketoacidosis; Atypical antipsychotics; Obesity; Dyslipidemia 
A NTIPSICÓTICOS DE SEGUNDA geração, incluindo clozapina $\left(\right.$ Leponex $\left.^{\circledR}\right)$, olanzapina $\left(\right.$ Zyprexa $\left.^{\circledR}\right)$, quetiapina $\left(\right.$ Seroquel $\left.{ }^{\circledR}\right)$, risperidona $\left(\right.$ Risperdal $\left.{ }^{\circledR}\right)$ e, mais recentemente, aripiprazol (Abilify ${ }^{\circledR}$ ) e ziprazidona (Geodon ${ }^{\circledR}$ ), são efetivos no tratamento da esquizofrenia, assim como os de primeira geração, como haloperidol, porém apresentando importante redução nos sintomas extrapiramidais (1-5). Entretanto, essas drogas têm sido relacionadas com significantes efeitos colaterais, como ganho de peso, distúrbios no metabolismo de lípides, intolerância à glicose e diabetes mellitus tipo 2 , podendo apresentar-se, ainda, como cetoacidose diabética, levantando questionamentos sobre os possíveis mecanismos envolvidos nessa associação, sugerindo a inclusão do endocrinologista na equipe multidisciplinar de cuidados aos pacientes com doenças psiquiátricas.

A seguir, apresentamos o caso de um paciente com cetoacidose diabética ao diagnóstico da doença, cerca de 1 mês após introdução da clozapina.

\section{RELATO DE CASO}

JFV, 28 anos, sexo masculino, natural e procedente de Belo Horizonte, com diagnóstico de esquizofrenia aos 18 anos, em uso por 10 anos de clonazepam, levomepromazina e biperideno, os quais foram suspensos devido ao desenvolvimento de distonia cervical tardia (laterocolo), sendo substituídas por clozapina, com aumento progressivo da dose, até $150 \mathrm{mg} /$ dia.
Duas semanas após início da medicação, ainda em fase de ajuste da dose, iniciou progressivamente com poliúria, polidipsia, prostração, emagrecimento acentuado (cerca de $15 \mathrm{~kg}$ ) e vômitos, sendo tais sintomas atribuídos inicialmente à descompensação da doença de base. Cerca de 1 mês após início da clozapina, foi admitido para internação no Serviço de Endocrinologia da Santa Casa de Belo Horizonte, proveniente de um pronto-socorro local, em cetoacidose diabética, com insulinoterapia e hidratação venosa, sem focos infecciosos e com medicação antipsicótica suspensa.

Paciente sem história familiar de diabetes mellitus, tireoidopatias ou outras patologias.

Seu IMC à admissão foi $24,2 \mathrm{~kg} / \mathrm{m}^{2}$. Os exames laboratoriais à admissão e durante a internação encontram-se na tabela 1 .

Recebeu alta hospitalar após 1 semana, em uso de insulina NPH 50 unidades/dia, suspensa após 14 dias. Paciente mantém-se atualmente, 5 meses após a internação, sem uso de medicações para diabetes, assintomático, com dieta livre e controle glicêmico satisfatório (glicemias de jejum 90/102/110 mg/dL; glicemias 2 horas pós-prandiais 100/120/108 mg/dL).

\section{DISCUSSĀO}

Há décadas, anteriormente à introdução das drogas antipsicóticas, estudos sugerem que a intolerância à glicose seja mais comum em pacientes com esqui-

Tabela 1. Exames laboratoriais durante a internação.

\begin{tabular}{lcc}
\hline Exames & Resultados & Valores de referência \\
PH & 7,21 & 7,32 a 7,43 \\
HCO3- & $13,1 \mathrm{mmoL} / \mathrm{L}$ & 22 a $29 \mathrm{mmoL} / \mathrm{L}$ \\
BE & $-13,4 \mathrm{mmoL} / \mathrm{L}$ & -2 a $+2 \mathrm{mmoL} / \mathrm{L}$ \\
Cetonemia & +++ & negativo \\
Glicemia & $350 \mathrm{mg} / \mathrm{dL}$ & 70 a $99 \mathrm{mg} / \mathrm{dL}$ \\
Potássio sérico & $4,5 \mathrm{mEq} / \mathrm{L}$ & 3,7 a $5,6 \mathrm{mEq} / \mathrm{L}$ \\
Uréia & $41 \mathrm{mg} / \mathrm{dL}$ & 10 a $40 \mathrm{mg} / \mathrm{dl}$ \\
Creatinina & $1,0 \mathrm{mg} / \mathrm{dL}$ & 0,8 a $1,3 \mathrm{mg} / \mathrm{dL}$ \\
Hemoglobina & $13,60 \mathrm{~g} / \mathrm{dL}$ & 14,4 a $17,6 \mathrm{~g} / \mathrm{dL}$ \\
Leucócitos & $9600 / \mathrm{mm}$ & 5000 a $10000 / \mathrm{mm}$ \\
Anti-GAD & $0,3 \mathrm{U} / \mathrm{mL}$ & $1,0 \mathrm{U} / \mathrm{mL}$ \\
Anti-IA2 & $0,4 \mathrm{U} / \mathrm{mL}$ & $0,5 \mathrm{U} / \mathrm{mL}$ \\
Anticorpo antimicrossomal & $<10 \mathrm{UI} / \mathrm{mL}$ & $<34 \mathrm{Ul} / \mathrm{mL}$ \\
TSH & $1,62 \mu U \mathrm{~mL}$ & 0,3 a $5,0 \mu \mathrm{ml} / \mathrm{mL}$ \\
Peptídeo C & $2,6 \mathrm{ng} / \mathrm{dL}$ & 1,1 a $5,0 \mathrm{ng} / \mathrm{dL}$ \\
HbA1C & $9,1 \%$ & 4,5 a $6,5 \%$ \\
Colesterol total & $127 \mathrm{mg} / \mathrm{dL}$ & Inferior a $200 \mathrm{mg} / \mathrm{dL}$ \\
Triglicérides & $205 \mathrm{mg} / \mathrm{dL}$ & Inferior a $150 \mathrm{mg} / \mathrm{dL}$ \\
\hline
\end{tabular}


zofrenia do que na população em geral $(6 \%$ e $1 \%$, respectivamente) (6), tendo um estudo italiano, incluindo 95 pacientes esquizofrênicos, encontrado uma prevalência de $15,8 \%$ de diabetes, independentemente do tratamento usado, comparada a $3 \%$ na população italiana em geral (7).

A partir de 1994, relatos na literatura psiquiátrica associam principalmente o uso da clozapina e da olanzapina ao diabetes e cetoacidose diabética, sendo a quetiapina associada a tais efeitos em menor proporção (8); já em relação à risperidona, a associação é menos consistente (9), parecendo igualar-se ao risco do tratamento convencional, não tendo sido relatado até o momento que ziprasidona e aripiprazol causem hiperglicemia.

Em relação à clozapina, uma revisão mostrou que cerca de $20 \%$ dos pacientes tratados demonstraram um aumento de, no mínimo, $10 \%$ no peso anterior ao início do tratamento, cuja duração variou de 12 semanas a 12 meses (10), com outro mostrando um ganho médio maior que $20 \%$ em relação ao basal, após 52 semanas (11).

Dois estudos mostraram uma correlação positiva entre níveis de insulina e concentração sérica da clozapina. Análise de 18 pacientes que receberam tratamento com clozapina por, no mínimo, 6 meses mostrou que 11 (61\%) tinham hiperinsulinemia em jejum e 13 (72\%) tinham níveis de peptídeo C elevado (12); Melkerson e Hulting evidenciaram níveis elevados de insulina em jejum em 7 entre 14 pacientes recebendo terapia, embora a média nos níveis de insulina tenha permanecido dentro dos limites normais (13).

A associação entre clozapina e diabetes tem sido bem documentada. Henderson e cols. apresentaram um estudo de 5 anos de seguimento, com 82 pacientes em uso de clozapina há pelo menos 1 ano, idade média de 36,4 anos e média de IMC de 26,9 , com $36,6 \%$ dos pacientes sendo diagnosticados com diabetes durante o tratamento. Embora tenha havido aumento significante do peso durante o estudo, este não foi associado com aumento do risco de diabetes nesta amostra, com 30 dos 82 pacientes desenvolvendo doença sem ganho de peso (14).

Hagg e cols. compararam 63 pacientes tratados com clozapina, com 67 em tratamento com neurolépticos convencionais, e encontrou no primeiro grupo uma incidência de $12 \%$ de diabetes tipo 2 e $10 \%$ de intolerância à glicose; no segundo grupo, as incidências foram 6 e 3\%, respectivamente. Pacientes recebendo clozapina foram significativamente mais jovens que o grupo controle, embora os dois grupos não tenham se diferenciado em relação a peso, IMC ou prevalência de diabetes em parentes de primeiro grau (15).
Dados epidemiológicos do FDA, entre janeiro de 1990 e fevereiro de 2001, identificaram 384 casos, sendo 323 definidos como hiperglicemia de início recente $(53 \%$ com diabetes definido pelos critérios atuais), 54 como exacerbação de diabetes preexistente e 7 casos não bem definidos. Entre os de início recente, a média de idade foi de $39 \pm 11$ anos, sendo cerca de $75 \%$ com idade inferior a 50 anos, abaixo da idade típica de início do diabetes tipo 2 . O tempo entre o início da terapia com clozapina e o surgimento do diabetes foi curto na grande maioria, ocorrendo dentro de 1 mês em $27 \%$ e 3 meses em $54 \%$ dos pacientes. Exacerbação do diabetes preexistente também ocorreu precocemente: em 1 mês para $38 \%$ e em 3 meses para $64 \%$ dos pacientes. Dados do seguimento demonstraram que, em $78 \%$ dos pacientes nos quais a clozapina foi suspensa, melhora do diabetes foi documentada. A severidade da hiperglicemia variou de intolerância leve a cetoacidose e coma hiperosmolar, com 51 pacientes apresentando-se ao diagnóstico com glicemias $\geq 700 \mathrm{mg} / \mathrm{dl}$, sendo cetoacidose diabética a forma de apresentação em 80 pacientes $(20,83 \%)$. Nenhuma evidência de obesidade ou ganho importante de peso, após início da terapia, ocorreu em 26\% dos pacientes (16).

Os fatores de risco associados à cetoacidose diabética não são bem entendidos. A raça parece ser um fator importante, com a grande maioria dos casos apresentando-se em afro-americanos e hispânicos; em relação à idade, a maioria se encontra entre os 30 e 50 anos, com proporção entre sexo masculino:feminino de 3:1 (17). Nos relatos de cetoacidose, a dose de clozapina variou entre 200 e $500 \mathrm{mg} / \mathrm{dia}$, com um episódio ocorrendo com dose de $150 \mathrm{mg} /$ dia (18).

Aumento nos níveis de triglicérides com clozapina tem sido documentado. Wirsching e cols. demonstraram um aumento de 34 a $42 \%$ nos níveis de triglicérides em 39 pacientes (19), com relatos da American Diabetes Association de que 56\% dos pacientes em tratamento apresentam níveis de triglicérides elevados (> $200 \mathrm{mg} / \mathrm{dl})$; já os níveis de colesterol total aumentaram em cerca de $5 \%$ em relação ao basal, com $4 \%$ de aumento no LDL e $6 \%$ de diminuição no HDL, sendo estas alterações no perfil lipídico comprovadas em outras séries, não estabelecendo relação com o ganho de peso $(20,21)$.

Assim como a clozapina, a olanzapina também associa-se a ganho de peso, com aumento em relação ao basal de 6-12 kg, em 6 a 12 meses de tratamento, com alguns estudos não demonstrando esta associação em até $24 \%$ dos pacientes $(22,23)$. Alterações nos níveis de insulina plasmática também foram observadas 
com olanzapina, com aumento significante na resistência à insulina comparando-se com os níveis basais (24), com dois estudos demonstrado níveis elevados de insulina em $31-71 \%$ dos pacientes tratados com essa droga $(13,25)$.

Entre 1994 e 2001, o FDA constatou 237 casos de diabetes ou hiperglicemia com olanzapina, 79\% sendo diabetes recém-diagnosticado e $19 \%$, exacerbação de diabetes preexistente (26). Entre os recémdiagnosticados, a média de idade foi 39,8 $\pm 12,4$ anos (68\% ocorrendo antes dos 45 anos), sendo esta $51,7 \pm$ 15,4 anos nos casos de exacerbação da doença. O tempo entre o início da terapia e o diagnóstico variou de 2 dias a 45 meses, $73 \%$ ocorrendo dentro de 6 meses ( $47 \%$ no primeiro trimestre); nos pacientes com doença agudizada, $84 \%$ dos eventos ocorreram nos primeiros 3 meses. Cetoacidose diabética foi um evento freqüente nesta série, ocorrendo em 80 dos $237 \mathrm{ca}$ sos $(33,8 \%)$. Pesquisas na Medline evidenciaram 33 relatos detalhando a associação de olanzapina e diabetes (27), com Ananth e cols., em uma recente revisão, encontrando dados semelhantes ao FDA em uma série de 26 casos. Cerca de $77 \%$ dos pacientes que descontinuaram a olanzapina recuperaram o controle glicêmico sem antidiabéticos orais ou insulina (28).

Estudos clínicos experimentais mostraram que a olanzapina tem efeito adverso importante no perfil lipídico, principalmente nos níveis de triglicérides, que aumentaram em cerca de 38\%, com mínimas alterações nos níveis de colesterol total (6\%) (19); Allison e cols. não observaram correlação entre ganho de peso e alterações no perfil lipídico (23).

Já em relação à risperidona, os poucos estudos até o momento não reproduzem os efeitos relacionados à clozapina e à olanzapina. $\mathrm{O}$ ganho de peso tem sido modesto, com média de $2 \mathrm{~kg}$, tanto a curto (cerca de 10 semanas) quanto a longo prazo ( 52 semanas), o que não parece estar relacionado à dose utilizada (23). Cento e trinta e um casos de diabetes ou hiperglicemia foram relatados pelo FDA entre 1993 e 2002, dos quais $60 \%$ com diabetes mellitus de início recente, com média de idade de $34,8 \pm 15,7$ anos, sendo esta de 48,8 $\pm 17,5$ anos na exacerbação de diabetes preexistente ( $35 \%$ dos casos). O tempo entre o início da terapia e a hiperglicemia variou de 1 dia a 48 meses, com $68 \%$ ocorrendo nos primeiros 6 meses. A risperidona foi suspensa em 47 pacientes e reduzida em cinco; os dados do seguimento destes pacientes foram escassos, embora melhora do controle glicêmico tenha sido observada em 12 desses pacientes (29).

Em contraste com o grande número de casos de cetoacidose associados à clozapina e à olanzapina, pouco se tem atribuído à risperidona. Croarkin e cols. relataram um caso de cetoacidose diabética em um paciente com infecção pelo HIV, com esta possivelmente contribuindo para o risco individual do paciente para o diabetes (30). Wilson e cols. relataram um caso de cetoacidose, ocorrendo 5 dias após o acréscimo de clozapina à risperidona (31). Gianfrancesco e cols., em um estudo incluindo pacientes não tratados $(\mathrm{n}=10296)$ ou em terapia antipsicótica $(\mathrm{n}=6582)$, sendo $1.675 \mathrm{com}$ risperidona, concluíram que o risco de desenvolvimento de diabetes atribuído a essa droga, usando análise de regressão logística, não foi diferente dos pacientes não-tratados (32). Analisados juntos, os estudos concluem que a risperidona não está associada a aumento consistente no risco de diabetes, o que poderia ocorrer no acréscimo dessa droga a tratamentos que levem ao aumento do peso e da adiposidade. Como essa medicação responde por $37 \%$ das prescrições de antipsicóticos de segunda geração nos Estados Unidos, os poucos casos relatados não podem ser atribuídos simplesmente ao uso limitado da droga. Os estudos até o momento sugerem, ainda, limitada e não consistente associação da risperidona com alterações no perfil lipídico (33).

A quetiapina foi introduzida em 1988 e os estudos em relação aos efeitos metabólicos são ainda iniciais. O ganho de peso parece pouco significante, de 2 a $5,5 \mathrm{~kg}$, com trabalhos mostrando ganho de até $7 \%$ do peso corporal inicial (34). O principal levantamento da associação ao diabetes foi apresentada pelo FDA, com dados de 1997 a 2002, apresentando 46 pacientes, $74 \%$ recém-diagnosticados, com média de idade de $31,2 \pm 14,8$ anos, e intervalo de tempo do início da terapia ao diagnóstico de 1 dia a 21 meses, com $41 \%$ ocorrendo nos primeiros 6 meses. Em 50\% dos pacientes nos quais a medicação foi descontinuada, houve melhora do controle glicêmico. Cetoacidose ocorreu em 21 pacientes (35); 23 novos casos, relatados pelos mesmos autores, em um adendo ao último trabalho, identificaram uma incidência de $34,8 \%$ de cetoacidose. Relatos de alterações no perfil lipídico são limitados e contraditórios, variando de hipertrigliceridemia severa $(>600 \mathrm{mg} / \mathrm{dl})(36,37)$ a diminuição de até $25 \%$ nesses níveis (19).

Ziprasidona e aripiprazol são relacionados a mínimos efeitos no peso corporal $(23,38)$, com dados até o momento não os associando com aumento do risco de diabetes ou dislipidemia (28).

Os mecanismos pelos quais os antipsicóticos atípicos causam distúrbios metabólicos não são completamente entendidos, principalmente porque a própria esquizofrenia tem um papel no desen- 
volvimento dessas alterações. Ganho de peso, como causa de resistência à insulina e, conseqüentemente, síndrome metabólica, são uma das explicações oferecidas, embora relatos discordando dessa associação sejam freqüentes. No mais, a suspensão da droga tem normalizado os níveis glicêmicos antes mesmo que a perda de peso seja aparente. Hiperglicemia, associada a hiperinsulinemia e níveis elevados de peptídeo C e leptina, relacionam-se, como já citado, principalmente à clozapina. Ardizzone e cols. encontraram uma correlação entre o uso da risperidona e da clozapina com inibição do transporte de glicose, em culturas de células PC12 (representantes de um modelo de diferenciação neuronal) e a indução de hiperglicemia in vivo (39). Pancreatite aguda é outro efeito relacionado, até o momento, principalmente à clozapina, seguida da olanzapina e risperidona, com relatos de cetoacidose concomitante ao desenvolvimento dessa patologia (40). Em relação ao mecanismo envolvido na cetoacidose diabética, apesar de ainda pouco entendido, parece estar relacionado a um efeito inibitório direto nas células beta pancreáticas, com casos de níveis de peptídeo $C$ entre 0,7 e $0,9 \mathrm{nmol} / \mathrm{dL}$ à admissão do paciente, com normalização após retirada da droga, sugerindo uma deficiência temporária de insulina (41).

Essas observações sugerem que a resistência à insulina não deve ser o único fator responsável pelo desenvolvimento do diabetes, com o rápido aparecimento da doença, em muitos pacientes, levantando questionamentos sobre um efeito direto e possivelmente tóxico das drogas antipsicóticas.

Levando-se em consideração que o estilo de vida dos indivíduos com doenças psiquiátricas seja um importante fator de risco para distúrbios metabólicos, haja visto que obesidade, dieta irregular, sedentarismo e altas taxas de alcoolismo e fumo são mais prevalentes que na população em geral, os crescentes conhecimentos sobre os efeitos dos antipsicóticos sobre esses fatores têm aumentado o interesse sobre os cuidados no screening e monitorização destes pacientes. Ressaltamos aqui a importância do seguimento continuado dos pacientes, mesmo após suspensão da droga, pois, como visto no caso relatado, após 5 meses da descontinuação da clozapina o paciente ainda apresentava glicemias de jejum superiores a 100 $\mathrm{mg} / \mathrm{dL}$. A American Diabetes Association e a American Psychiatric Association publicaram, em 2004, um consenso para acompanhamento desses pacientes (42), com recomendações para screening incluindo IMC, pressão arterial, perfil lipídico e glicêmico, história pessoal e familiar de obesidade, diabetes, dislipidemia, hipertensão e doença arterial coronariana. An- tipsicóticos de segunda geração, com baixa propensão para ganho de peso e intolerância à glicose, devem ser considerados para pacientes com diabetes ou aumento do risco para a doença. Monitorização do peso deve ser realizada com 4,8 e 12 semanas após início ou substituição da terapia antipsicótica, e, depois, em visitas trimestrais. Medidas de glicemia e lípides são recomendadas 3 meses após início da terapia e, então, anualmente e a cada 5 anos, respectivamente, a menos que os riscos iniciais ou novos fatores indiquem monitorização mais freqüente (embora as recomendações do NCEP ATP III sejam de monitorização anual do perfil lipídico em pacientes com riscos para dislipidemia) (43).

A introdução de monitorização clínica regular, realizada por especialistas envolvidos no manuseio das drogas citadas e de seus possíveis efeitos colaterais, deve permitir uma detecção precoce de mudanças nesses importantes fatores de risco, melhorando globalmente o estado de saúde desses pacientes a longo prazo, evitando as conseqüências que os distúrbios metabólicos podem acrescentar ao futuro dos mesmos.

\section{REFERÊNCIAS}

1. Kane J, Honigfeld G, Singer J, Meltzer H. Clozapine for the treatment-resistant schizophrenic: a double-blind comparison with chlorpromazine. Arch Gen Psychiatry 1988;45:789-96.

2. Tollefson GD, Beasley CM Jr, Tran PV, Street JS, Krueger JA, Tamura RN, et al. Olanzapine versus haloperidol in the treatment of schizophrenia and schizoaffective and schizophreniform disorders: results of an international collaborative trial. Am J Psychiatry 1997;154:457-65.

3. Small JG, Hirsch SR, Arvanitis LA, Miller BG, Link CG. Quetiapine in patients with schizophrenia: a high- and low-dose double-blind comparison with placebo: Seroquel Study Group. Arch Gen Psychiatry 1997;54:549-57.

4. Marder SR, Meibach RC. Risperidone in the treatment of schizophrenia. Am J Psychiatry 1994;151:825-35.

5. Goff DC, Posever T, Herz L, Simmons J, Kletti N, Lapierre K, et al. An exploratory haloperidol-controlled dose-finding study of ziprasidone in hospitalized patients with schizophrenia or schizoaffective disorder. J Clin Psychopharmacol 1998;18:296-304.

6. Ward Jl. Glucose tolerance in schizophrenia. Orthomol Psychiatry 1972;44:137-40.

7. Mukherjee S, Decina P, Bocola V, Saraceni F, Scapicchio PL. Diabetes mellitus in schizophrenic patients. Compr Psychiatry 1996;37:68-73.

8. Takahashi M, Ohishi S, Katsumi C, Moriya T, Miyaoka H. Rapid onset of quetiapine-induced diabetic ketoacidosis in an elderly patient: a case report. Pharmacopsychiatry 2005;38(4):183-4.

9. Haupt DW, Newcomer JW. Risperidone-associated diabetic ketoacidosis. Psychosomatics 2001;42(3):279-80.

10. Iqbal MM, Rahman A, Husain Z, Mahmud SZ, Ryan WG, Feldman JM. Clozapine: a clinical review of adverse effects and management. Ann Clin Psychiatry 2003;15(1):33-48.

11. Bustillo JR, Buchanan RW, Irish D, Breier A. Differential effect of clozapine on weight: a controlled study. Am J Psychiatry 1996;153(6):817-9. 
12. Melkersson KI, Dahl ML. Relationship between levels of insulin or triglycerides and serum concentrations of the atypical antipsychotics clozapine and olanzapine in patients on treatment with therapeutic doses. Psychopharmacology (Berl) 2003;170(2):157-66.

13. Melkersson KI, Hulting AL. Insulin and leptin levels in patients with schizophrenia or related psychoses - a comparison between different antipsychotic agents. Psychopharmacology (Berl) 2001;154:205-12.

14. Henderson DC, Cagliero E, Gray C, Nasrallah RA, Hayden DL, Schoenfeld DA, et al. Clozapine, diabetes mellitus, weight gain, and lipid abnormalities: a five-year naturalistic study. Am J Psychiatry 2000;157:975-81.

15. Hagg S, Joelsson L, Mjorndal T, Spigset O, Oja G, Dahlqvist $R$. Prevalence of diabetes and impaired glucose tolerance in patients treated with clozapine compared with patients treated with conventional depot neuroleptic medications. J Clin Psychiatry 1998;59:294-9.

16. Koller E, Schneider B, Bennett K, Dubitsky G. Clozapine-associated diabetes. Am J Med 2001;111:716-23.

17. Wirshing DA, Spellberg BJ, Erhart SM, Marder SR, Wirshing WC. Novel antipsychotics and new-onset diabetes. Biol Psychiatry 1998;44:778-83.

18. Lafayette JM, Pirl WF, Henderson DC. Low-dose clozapine and diabetic ketoacidosis. Psychosomatics 2003;44(3):249-52.

19. Wirshing DA, Boyd JA, Meng LR, Ballon JS, Marder SR, Wirshing WC. The effects of novel antipsychotics on glucose and lipid levels. J Clin Psychiatry 2002;63:856-65.

20. Gaulin BD, Markowitz JS, Caley CF, Nesbitt LA, Dufresne RL. Clozapine-associated elevation in serum triglycerides. Am J Psychiatry 1999;156:1270-2.

21. Spivak B, Lamschtein C, Talmon $Y$, Guy N, Mester R, Feinberg $\mathrm{I}$, et al. The impact of clozapine treatment on serum lipids in chronic schizophrenic patients. Clin Neuropharmacol 1999;22:98-101.

22. Gupta S, Droney T, Al-Samarrai S, Keller P, Frank B. Olanzapine: weight gain and therapeutic efficacy. J Clin Psychopharmacol 1999;19(3):273-5.

23. Allison DB, Mentore JL, Heo M, Chandler LP, Cappelleri JC Infante MC, et al. Antipsychotic-induced weight gain: a comprehensive research synthesis. Am J Psychiatry 1999;156:1686-96.

24. Ebenbichler CF, Laimer M, Eder U, Mangweth B, Weiss $E$, Hofer $A$, et al. Olanzapine induces insulin resistance: results from a prospective study. J Clin Psychiatry 2003,64:1436-9.

25. Melkersson KI, Hulting AL, Brismar KE. Elevated levels of insulin, leptin, and blood lipids in olanzapine-treated patients with schizophrenia or related psychoses. J Clin Psychiatry 2000;61:742-9.

26. Koller EA, Doraiswamy PM. Olanzapine-associated diabetes mellitus [review]. Pharmacotherapy 2002;22:841-52.

27. Second-Generation (Atypical) Antipsychotics and Metabolic Effects. A Comprehensive Literature [review]. CNS Drugs 2005;19(suppl 1):1-93.

28. Ananth J, Venkatesh R, Burgoyne K, Gunatilake S. Atypical antipsychotic drug use and diabetes. Psychother Psychosom 2002;71:244-54.

29. Koller EA, Cross JT, Doraiswamy PM, Schneider BS. Risperidone-associated diabetes mellitus: a pharmacovigilance study. Pharmacotherapy 2003;23(6):735-44.

30. Haupt DW, Newcomer JW. Risperidone-associated diabetic ketoacidosis. Psychosomatics 2001;42(3):279-80.

31. Wilson DR, D'Souza L, Sarkar N, Newton M, Hammond C. New-onset diabetes and ketoacidosis with atypical antipsychotics. Schizophr Res 2002;59:1-6.
32. Gianfrancesco F, White R, Wang RH, Nasrallah HA. Antipsychotic-induced type 2 diabetes: evidence from a large health plan database. J Clin Psychopharmacol 2003,23(4):328-35.

33. Koro CE, Fedder DO, L'Italien GJ, Weiss S, Magder LS, Kreyenbuhl J, et al. An assessment of the independent effects of olanzapine and risperidone exposure on the risk of hyperlipidemia in schizophrenic patients. Arch Gen Psychiatry 2002;59:1021-6.

34. Borison R, Arvanitis LA, Miller BG. Atypical antipsychotic: efficacy and safety in a multicenter, placebo-controlled trial in patients with schizophrenia. J Clin Psychopharmacol 1996;16(2):158-69.

35. Koller EA, Weber J, Doraiswamy PM, Schneider BS. A survey of reports of quetiapine-associated hyperglycemia and diabetes mellitus. J Clin Psychiatry 2004;65(6):857-63.

36. Meyer JM. Novel antipsychotics and severe hyperlipidemia. J Clin Psychopharmacol 2001;21:369-74.

37. Domon SE, Cargile CS. Quetiapine-associated hyperglycemia and hypertriglyceridemia [letter]. J Am Acad Child Adolesc Psychiatry 2002;41:495-6.

38. Marder SR, McQuade RD, Stock E, Kaplita S, Marcus R, Safferman $A Z$, et al. Aripiprazole in the treatment of schizophrenia: safety and tolerability in short-term placebo-controlled trials. Schizophr Res 2003;61:123-36.

39. Ardizzone TD, Bradley RJ, Freman AM, Dwyer DS. Inhibition of glucose transport in PC12 cells by atypical antipsychotic drugs risperidone and clozapine and structural analogs of clozapine. Brain Res 2001;27(1-2):82-90.

40. Koller EA, Cross JT, Doraiswamy PM, Malozowski SN. Pancreatitis associated with atypical antipsychotics: from the Food and Drug Administration's MedWatch surveillance system and published reports. Pharmacotherapy 2003;23(9):1123-30.

41. Rigalleau V, Gatta B, Bonnaud S, Masson M, Bourgeois ML, Vergnot $V$, et al. Diabetes as a result of atypical anti-psychotic drugs $-a$ report of three cases. Diabet Med 2000;17(6):484-6.

42. American Diabetes Association; American Psychiatric Association; American Association of Clinical Endocrinologists; North American Association for the Study of Obesity. Consensus development conference on antipsychotic drugs and obesity and diabetes. Diabetes Care 2004;27(2):596-601.

43. National Cholesterol Education Program. Executive summary of the Third Report of the National Cholesterol Education Program (NCEP) Expert Panel on Detection, Evaluation and Treatment of High Blood Cholesterol in Adults (Adults Treatment Panel III). JAMA $2001 ; 285: 2486-97$.

\section{Endereço para correspondência:}

Janice Sepúlveda Reis

Centro de Estudos e Pesquisa da Clínica de Endocrinologia e Metabologia (CEPCEM)

Av. Francisco Sales $1111,5^{\circ}$ andar, ala D

30150-221 Belo Horizonte, MG

Fax: (31) 3213-0836

E-mail: janicesepulveda@terra.com.br 